\title{
Language retrogenesis as a window to language evolution
}

Antonio Benítez-Burraco ${ }^{1}$ and Olga Ivanova ${ }^{2}$

1. Department of Spanish, Linguistics and Theory of Literature (Linguistics), Faculty of Philology, University of Seville, Seville, Spain (email: abenitez8@ us.es)

2. Spanish Language Department, Faculty of Philology, University of Salamanca, Salamanca, Spain

\begin{abstract}
Several structurally simplified forms of language (sometimes called, 'degraded') have been explored as possible proxies to previous stages in the evolution of language. In this paper, we focus on two of such forms, namely, child language and language in aging. Specifically, we re-examine the hypothesis of language retrogenesis, according to which language loss in aging parallels, though inversely, language acquisition by the child. We present evidence supporting this view, particularly, in the domain of complex aspects of language with high cognitive reliance, including lexical access (demanding the mapping of meanings to phonological representations), the understanding of non-literal meanings (demanding the access to the context and to encyclopedic knowledge), the resolution of lexical competition, and the understanding and production of complex syntactic structures (demanding more working memory resources). We further consider whether these changes recapitulate aspects of language phylogeny in the species. We have also found some support to this view, as the involved areas (like the inferior frontal gyrus), are phylogenetically newer, as shown by interspecies comparison. Finally, we advance a new testing arena for this hypothesis, involving some of the cortico-subcortical circuits controlling both reactive aggression and complex syntax.
\end{abstract}

Keywords: language evolution; language retrogenesis; aging brain; child language development; complex syntax; lexical competence; self-domestication 


\section{Introduction}

Language is perhaps the most distinctive feature of the human condition. Since language does not fossilize, we need to rely on indirect evidence for inferring how human languages were in the past and how our ability to learn and use them evolved in our species. There have been multiple efforts to shed light on human language faculty (Fitch 2017) and one of the most interesting, yet less explored source of evidence concerns what Bickerton (1990) and Jackendoff (1999) labelled as 'degraded'forms of language. This term is used to refer to different types of structurally simplified and functionally limited linguistic systems, which may be found in either speakers with language deficits resulting from developmental or acquired disorders, or in speakers with an incomplete proficiency in a language that is being acquired as a first language or learned as a second language. The term also encompasses language systems with limited expressive possibilities, mostly aimed to serve as lingua franca. Despite certain unclarity on where to trace limits to the so-called 'degraded' forms of language, as the competence in a language (and the complexity of a language) is, to some extent, a matter of degree, one may assume that among such we should include any linguistic system lacking some/most of the structural features found in natural languages and that do not allow for complete functional communication. Accordingly, candidates to 'degraded' forms of language (and thus to proxies of previous stages in the evolution of language(s)) can include i) pathological instances of a language, either resulting from ontogenetic damage or acquired impairment; ii) pidgin languages and other restricted languages; iii) deaf children homesigns in non-signing families; iv) (initial stages in the development of) child language; v) subordinate/heritage languages in unbalanced bilinguals; and vi) L2 learners' interlanguages. Botha (2006) have discussed the utility of these linguistic systems for language evolution studies, whereas other authors have raised particular concerns about their specific types (see e.g. Slobin 2005 on child language as a 'degraded' form of language). Over the years, new potential candidates for 'degraded' forms of language have appeared. For instance, one could consider as such the initial stages in the process of language learning by computational models/artificial intelligence (Steels and Szathmáry 2018). In sum, being a catchy cover term for a set of interesting linguistic phenomena, this is an area of language evolution studies that needs to be revisited, and particularly, reexamined under the light of available evidence in different domains. 
One interesting finding about 'degraded' forms of language is that, perhaps surprisingly, they share many structural properties. For instance, the so-called "Basic Variety" in which many L2 learners fossilize (Klein and Perdue 1997) features many of the structural properties found in pidgins, including the absence of inflectional morphology or subordination. At the same time, these are properties that are acquired later by the child (see Luinge et al. (2006) for the order of milestones in child language development) and that are easily lost in e.g. patients with different aphasia syndromes (see Clark and Cummings (2003) for a global discussion of language abnormalities in different types of aphasia) or elder people with Alzheimer's disease (see Emery (2000) for a model of language decline following a hierarchical pattern). As a consequence, some aspects of language structure and use can be hypothesized to be more complex, and perhaps less foremost than others. More complex aspects would be then acquired later, and/or with more effort, and/or in the proper learning/usage environment only. More complex aspects would be also processed less efficiently and/or demand more cognitive/brain resources. Finally, more complex aspects would be more sensitive to damage, during acquisition or in the adult state. Moving to the domain of evolutionary linguistics, the common features exhibited by these 'degraded' forms of language have been equated, as noted, to the putative features exhibited by the (proto)languages spoken by our species in our remote past and/or by other hominin species in our lineage. For instance, Code (2011) based on his studies on non-fluent aphasia resulting from damage in left inferior frontal brain areas to suggest that the lexical speech automatisms of patients could mimic a pre-full syntax stage in language evolution. Code also argued that the recovery of aphasic speech parallels (early stages of) language evolution in our species. Likewise, Ardila (2006, 2015) took language changes due to aphasia as a basis for his hypothetization on language origin, suggesting a two-scale evolution of human language: selectional, or lexicalsemantic (as inspired by Wernicke's aphasia), and sequential, or grammatical (as inspired by Broca's aphasia). Underlying this sort of hypotheses is the view that language evolves from simpler to more complex forms and that complex language demands more sophisticated cognitive and behavioral abilities to be mastered, that only appeared fullfledged in our species. In their seminal paper on the Basic Variety, Klein and Perdue (1997) disagreed with the idea that the Basic Variety could be assigned to a protolanguage distinct from "fully-fledged" languages, but acknowledged that it exhibits the core attributes of the human language capacity, suggesting that the missing features could be regarded as secondary and thus, as appeared later. 
The possibility that these 'degraded' forms of language are real atavisms is controversial. True atavisms do exist in biology (see Hall (2010) for discussion), and real reversions to ancestral states can occur, but only if ancestral developmental programs are preserved (but silenced) in the derived population (Cabej 2012). Interestingly, candidate genes for conditions like schizophrenia (SZ) o autism spectrum disorder (ASD), both entailing language deficits impacting features that are absent in 'degraded' forms of language, like inflectional morphology, are enriched in genes that have been positively selected in our species (Srinivasan et al. (2016) or Polimanti et al. (2017), respectively). This might qualify the nature of the language problems observed in these conditions, that are both structural and functional, as truly atavistic, although some caution is in order (see BenítezBurraco and Boeckx (2014) for discussion). The same can be said of developmental regressions as found in conditions like ASD (Stefanatos, 2008) or epileptic disorders (Scheffer et al. 2006; Saraf et al. 2020). Underlying this view is not just that language evolves from simple to complex, as noted, but that recently evolved aspects of the human cognitive and behavioral phenotype, seemingly accounting for the more complex aspects of language, are more sensitive to damage, either developmental or acquired, because of the reduced resilience of the neuronal networks supporting them (see Toro et al. (2010) for ASD; Pattabiraman et al. 2020). That said, because this kind of disorders results for some type of damage, either genomic (developmental language disorders) or neurobiological (acquired language disorders), it might be difficult to differentiate between truly atavistic features and maladaptive symptoms resulting from damage. In the first case, what we have is a brain that is developing differently in order to accommodate some genetic alteration. In the second case, what we have is a neurotypical brain that has to accommodate a neurological damage occurred once it was normally configured. Putting it differently, one has to be cautious about the assumption that these pathological losses of function really mirror the putative gain of function occurred in our ancestors.

By these reasons, in our paper, we have focused on language changes associated to neurotypical development with the aim of supporting the use of specific stages in the acquisition/loss of language as proxies or windows to the evolution of language. Specifically, we will re-examine the retrogenesis hypothesis of language decline during aging (Reisberg et al. 1999), according to which language changes in old age mirror the changes occurred during language acquisition by the child. Healthy aging itself is not 
frequently associated with a clear language impairment, but rather with cognitive (and language) readjustment for successful language processing and production (Peelle 2019). Still, some changes due to age-related cognitive decline are observed. More generally, ontogeny (even abnormal ontogeny) does not exactly recapitulate phylogeny. However, when these putative linguistic proxies of early language evolution are considered together with evolutionary changes in brain anatomy and function, more robust findings can be achieved, as shown by some recent works on e.g. expletives and derogatory language (Progovac 2015; Benítez-Burraco and Progovac 2021). Accordingly, we wish to examine the possibility that these specific 'degraded' forms of language (i.e. last stages of language development in the child vs. early stages of language decline in the old adult) could help us reconstruct several important aspects of early (proto)languages. In doing this, we will heavily rely on neurobiological and genetic evidence. As noted by Pattabiraman and colleagues (2020: 91), our species-specific cognitive abilities arose in part from changes in the organization and function of preexisting neural devices, and these changes account for distinctive aspects of our neurodevelopment, but also for our predisposition to suffer from neurodegenerative diseases.

The paper is structured as follows. First, we provide a summary of current findings about language change in healthy aging. Second, we review (and update) the retrogenesis theory, by comparing language changes in healthy aging with language acquisition by the child. Third, we provide a succinct comparison between brain changes in the child during acquisition and brain changes in the aging during involution, with the aim of knowing whether language acquisition also parallels, though inversely, language decay at the neurobiological level. Fourth, we will discuss our results in the light of current theories about the evolutionary changes in neuronal substrate of language. This will lead us to reconsider, once again, the idea that language ontogeny recapitulates language phylogeny. But we will do this from an EvoDevo perspective, according to which evolution is fueled by changes in developmental processes, that is, ontogeny creates phylogeny. We expect this approach to enable us to properly qualify the claim that language loss in healthy aging can be regarded an informative proxy or window to specific stages of language evolution. 


\section{Cognitive and language changes in healthy aging}

Cognitive decline in healthy aging inevitably leads to age-related language changes, that progress from less-to-more impairment at all levels of language structure (summarized in Figure 1). The reason is that aging impacts on abilities that are crucially involved in language processing, like executive function, attention, learning ability, memory, or concentration. Some evidence exists of language preservation in senescence (see Cohen et al. 2019; Pistono et al. 2021), but experimental differences aside, this apparent preservation may result from the greater irregularity/variability in the degree of affection of different cognitive functions observed in older adults (Harada et al. 2013; Leal and Yassa 2019). Language alterations in typical aging are thus suggested to be due to the changes in brain circuits supporting broad cognitive functions, although a disruption of the speech-language network itself may also exist, and we will discuss it in section 4 below.

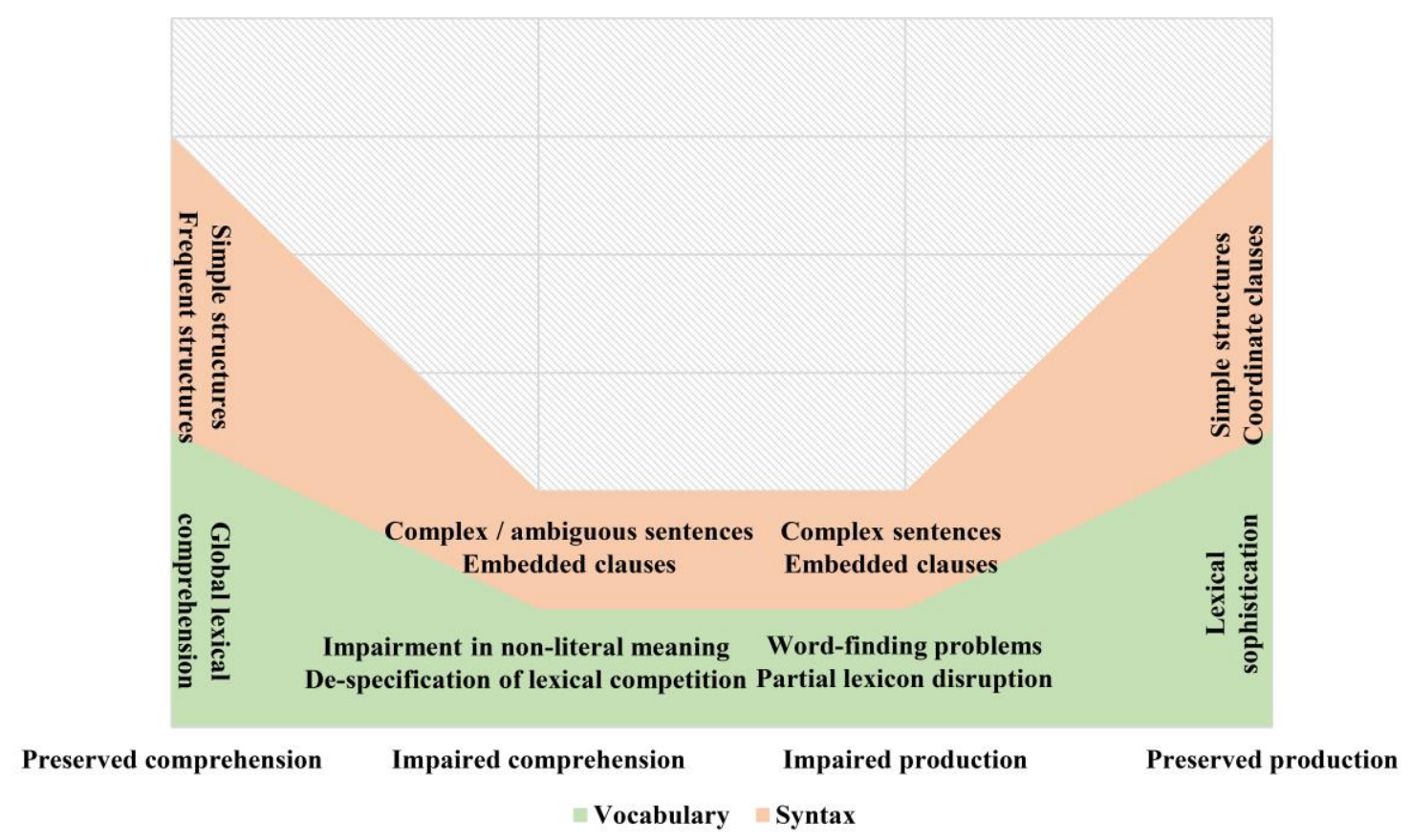

Figure 1. Preserved and impaired language functions in healthy aging in the domains of syntax (in salmon) and the lexical semantics (in green). As noted, healthy aging conveys to difficulties in comprehension and production of complex sentences and sentences with embedded clauses (middle), while comprehension of simple and frequent syntactic structures (left) and production of simple and coordinate clauses (right) are preserved. Likewise, healthy aging conveys to impaired comprehension of non-literal meanings and de-specification of lexical competition and word-finding problems during production (middle), while global lexical comprehension (left) and production (right) are preserved. 
Among the cognitive functions that can account for language impairment in aging one finds working memory, cognitive processing, and inhibitory control (Abrams and Farrell 2010). Motor execution also declines with age, and this circumstance seemingly contributes to explain speech changes during senescence (Tremblay et al. 2018), although anatomical shifts impacting on aspects like voice quality, speed and pitch also account for differences (Tucker et al. 2020). The evolutionary history of speech is intriguing and an important chapter in any account of the evolution of language (e.g. Fitch 2000; de Boer 2019). However, in our paper we will focus, as noted, on the cognitive aspects of language. By a similar reason, we are not addressing here changes in patterns of language use (i.e. pragmatics) during aging (but see Bambini et al. (2021) for an interesting discussion around the cognitive and pragmatic changes during aging).

A very rough picture of language changes in healthy aging suggests that there exists a dissociation between disrupted language production, on the one hand, and (apparently) preserved language comprehension, on the other hand (Burke and Mackay 1999; Abrams and Farrell 2010). Also, a correlation seems to exist between the complexity of the language task and its temporal order for disruption, with problems in language production predating changes in language comprehension. At both levels, lexical and syntactic aspects result significantly more disrupted. We now provide a more detailed depiction of these changes.

Language production. As noted, language production is the primary affected language domain in aging, with phono-lexical (word access and production) and syntactic (complex sentences) levels being the most affected.

Lexical productive difficulties in aging are mainly framed withing the global phenomenon of word-finding. Healthy elderly is reported to impair on accessing mental lexicon as measured through naming (Melvold et al. 1994), to exhibit word-find difficulties deriving in frequent circumlocutions (Glisky 2007) and to present tip-of-thetongue problems during word retrieval (Burke and Mackay 1999). Difficulties are particularly notable for accessing and producing proper names (Ouyang et al. 2020), mainly due to their specific semantic structure against common words (Juncos-Rabadán et al. 2010). Among common names, problems are initially observed with low-frequency words and words with long-term inactivity due to insufficient priming and poorer 
semantic connections (Barresi et al. 2000), suggesting that more complex and less semantically connected words are among first candidates to loss in healthy aging. Overall, problems for word-finding in aging are suggestive of a general difficulty for accessing and recovering word-forms (Burke et al. 2000). Experimental evidence points to a disruption in the elderly's capacity to adjust lexical concept to its phonological form (Burke and Mackay 1999; Barresi et al. 2000; Tremblay et al. 2018). This would explain language production deficits, like dysfluencies, tendency towards word repetition and filled pauses, as well as phoneme omission (Burke and Graham 2012), but also the observed positive effect of phonological priming (Ouyang et al. 2020) and context facilitation effects (Burke et al. 2000) on word retrieval in aging. These word-finding problems seem to boil down to neurobiological changes in the elder brain. Accordingly, tip-of-the-tongue effects have been hypothesized to result from reduced gray matter in left insula, known to be implicated in phonological production (Shafro et al. 2007). Likewise, difficulties have been related to shrinkages in the left prefrontal cortex and temporo-occipital regions, with consequent increases in insular regions activity (Manenti et al. 2013), and/or to a reduction in dopaminergic and cholinergic activity in selected areas of the brain (Albert 1991). At the same time, older adults feature an extremely extensive vocabulary, as vocabulary size increases during the lifespan (Glisky 2007; Gollan and Goldrcik 2019). They also exhibit advanced lexical sophistication and semantic expertise (Spreng and Turner 2019), deeply relying on crystallized knowledge (Rabaglia and Salthouse 2011), which have been argued to compensate for greater demands in cognitive control in aging.

In the domain of syntax, older adults generally tend to produce shorter and less complex sentences. Age conveys preference for coordinate and right-branching syntactic constructions (Kempler et al. 2001) and an avoidance of left-branching stems and propositionally dense semantic content (Rabaglia and Salthouse 2011). Simpler syntactic structures in aging include more vague terms and more empty pauses (Shafro and Tyler 2014). These differences have been hypothesized to result from age-related limitations in the working memory system (Kempler et al. 2004), and generally, lower memory demands (Venneri et al. 2018), seemingly boiling down to decreased functional connectivity in syntax-supporting neural networks, mainly in left pars opercularis (Antonenko et al. 2013). Lexical difficulties, of the sort highlighted above, play an important role in syntactic simplification in aging. Elderly speakers maintain their ability 
to follow syntactic priming and lexical boost, proving to maintain syntactic representations that underly sentence production with aging (Hardy et al. 2017). They also systematically rely on syntactic facilitation effects and on the context (Shafro and Tyler 2014; Hardy et al. 2020). This is why some authors have suggested that syntactic deviations in healthy aging may be explained mostly by lexical problems, rather than by a specific impairment of syntactic processes involved in sentence production (Hardy et al. 2020).

Language comprehension. As noted, when compared to language production, language comprehension abilities show longer resistance to aging effect and, thus, better preservation, seemingly because of their spared linguistic knowledge and command for procedural rules despite other cognitive declines (Wingfield et al. 2015). However, they also exhibit difficulties in diverse domains.

Regarding the lexical competence, vocabulary comprehension is not affected entirely, but there are rather specific aspects of lexical integration that parallel what we find in the domain of production and that result in two specific deficits: lexical competition and difficulty to recognize non-literal meanings. The former implies that olders are more prone to activate neighboring non-target words, so that while accessing frequently-used words remains stable, accessing infrequent words and words with many phonological neighbors disrupts (DeDe and Knilans Flax 2016). Moreover, they gradually lose the ability to contextually predict during comprehension and as a consequence, they typically change to less predictive modes of comprehension (Wlotko et al. 2010). The fact that in noisy contexts older speakers disrupt significantly more in word comprehension (Tucker et al. 2020) also supports the view that aging results in a de-specification for lexical processing. Overall, these differences have been explained in terms of an age-related general inhibition deficit, that results in lower inhibitory control of lexical information (Mattys and Scharenborg 2014). In addition, older speakers lose the ability to infer and comprehend complex and non-literal meanings, this impacting negatively on the capacity for resolving lexical ambiguity (Lee and Federmeier 2010) and comprehending conventional metaphors (Sundaray et al. 2018) or proverbs (Uekermann et al. 2008).

Syntactic comprehension in healthy aging somehow parallels vocabulary comprehension disruption. In general, older adults show difficulty in retaining and understanding 
sentences with complex syntactic structures and ambiguous syntactic conditions (Abrams and Farrell 2010). Demanding sentences, like those containing long-distance dependencies or embedded clauses, can imply longer online processing for older speakers and eventually, result in comprehension problems (Wingfield and Grossman 2006; Caplan et al. 2011). Sentences with less frequent syntactic order are also candidates to comprehension disruption (DeDe and Knilans Flax 2016). Older adults can even find it difficult to comprehend simple sentences or sentences with agreement errors (Poulisse et al. 2019).

Overall, arising difficulties for language comprehension in aging have been linked to agerelated working memory limited capacity (Wingfield and Grossman 2006), seemingly resulting from alterations in prefrontal (Uekermann et al. 2008) and frontal cortex (Lee and Federmeier 2010), supporting working memory and inhibitory control. At the same time, the higher preservation of language comprehension abilities in healthy elderly may be due to the higher neuronal burden of production (Diaz et al. 2016), as well as to the increased reliance of the elderly on the broader context of language input during comprehension compared to what happens in production (Shafro and Tyler 2014).

\section{Language acquisition and language decline vis-a-vis}

The hypothesis of retrogenesis was initially defined by Reisberg and colleagues (1999) and suggests that changes in aging follow the principle of 'last in, first out'. According to this view, the progressive cognitive decline in aging reverses the order of acquisition of the same functions by children. To some extent, this hypothesis has been confirmed by clinical data (Rubial-Álvarez et al. 2013). As discussed in the previous section, complex aspects of language become impaired with age. At the same time, child language acquisition follows a 'from the simplest to the most complex' pattern. Consequently, one could assume that language also adheres to the retrogenesis principle, with initial stages in language decline in healthy aging pointing at last stages of language development, and vice versa. As with general cognition, evidence supporting this view comes mostly from the domain of clinical linguistics. Hence, some sort of retrogenetic involution has been observed in Alzheimer's disease with regards to lexical-semantic abilities (Simoes Loureiro and Lefebvre 2016; Gayraud et al. 2019), naming performance (Kim et al. 2011) 
and semantic categorical association (Mello et al. 2008). By contrast, to the best of our knowledge, language retrogenesis has not been yet explored for healthy aging.

Children acquire more simple language tasks first. Language comprehension precedes language production in all domains (Vesser-Bochane et al. 2020). Among such earlyacquired language functions is word comprehension (Messer 2015), which precedes expressive vocabulary acquisition (Roemer et al. 2019). Simple syntax understanding precedes simple syntax production, as well as complex syntax comprehension and production (Huttenlocher et al. 2002). Moreover, the complexity of the vocabulary acquired at each stage is a robust predictor of complex grammar development (Dixon and Marchman 2007). In general, as summarized by Bates and Dick (2002), language acquisition by the child proceeds through distinctive, although partially overlapping stages: word comprehension (8-10 months), word production or naming (11-13 months), word combinations (18-20 months), grammaticization (24-30 months), and acquisition of simple syntactic structures, including sentence types (i.e., declarative, interrogative, imperative, etc.) (30-34 months). Although more complex syntax can be observed at younger ages (Vasilyeva et al. 2007), longer sentences and more variable syntactic constructions are usually ascribed to later ages, starting from age 5 (Tolchinsky 2004). Lexical development does not reach adult-like structure and functionality, specifically automatization, until 7-to-9 years old, when the number of stored words is enough to create sophisticated networks encompassing semantic and phonological relations. In this respect, child language development begins from simple, coordinate and non-related understanding and production of language structures, progressively converting to more complex and dependent structures with multiple functions.

In Table 1 we summarize language acquisition milestones in contrastive perspective with language changes in aging. Still, some caution is in order. Language development is relatively easy to trace chronologically, seemingly because basic aspects of cognitive development are stably associated with biological developmental stages. By contrast, associating age-related language changes to specific lifetime stages is more difficult, as older adults show heterogeneous cognitive reserves and different decline paces (see Kempler et al. 2001). 


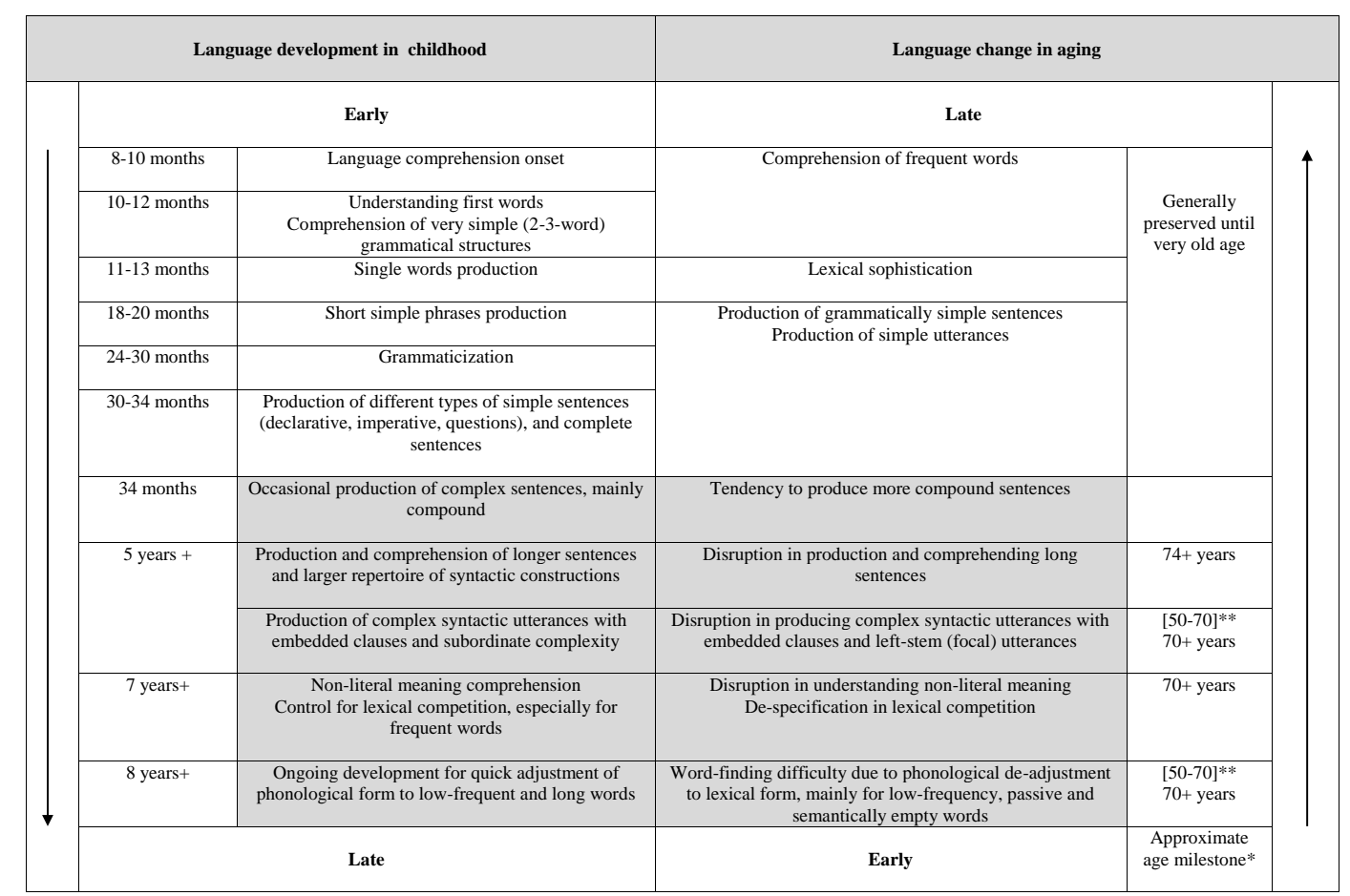

Table 1. Language acquisition in neurotypical childhood and language impairment in healthy aging: chronological stages and associated phenomena. In clear gray we highlight coincident late-childhood/early-aging matching stages.

*We assume the extreme difficulty to assign language changes in aging to any specific chronological milestones, mainly because of high inter-subject variability. This column, thus, is essentially speculative, though based on real data proceeding from different experimental studies. **The change itself is long-lasting and progressive. The end age means the most disruptive point for language change.

In what follows, we provide a more detailed comparison between the aspects of language known to decline in healthy aging (as described in Section 2) and the corresponding aspects in language acquisition stages, with the aim of providing additional support to the retrogenesis hypothesis with regards to language.

Deficits in lexical access during production due to phonological disruption. As noted in Section 2, one of the first changes in healthy aging refers to word-retrieval difficulties due to constraints in adjusting phonological forms to lexical concepts. During language development, children mainly owe word-finding difficulties to imprecise phonological representation of words (Messer and Dockrell 2006), similarly to older speakers. Likewise, children from 8 to 10 years old exhibit tip-of-the-tongue problems when trying to access low-frequent and long words, as well as words from sparse neighborhoods (Newman et al. 2018): this parallels the observed in elderly. Considering age occurrence, 
age-related impairment of lexical access reverses the principles of lexical access in children.

De-specificiation and literality in word comprehension. Children's ability to not underor over-generalize word meaning is a late-acquired language function, with major development after age 5 (Tolchinsky 2004). Prior to that age, they frequently over-extend words. Indeed, initially children prefer semantic, rather than contextual source for interpreting words and utterances (Ryder and Leinonen 2013) and they usually need several years of development and exposure to language use in order to develop the ability to grasp non-literal or not-given meaning. Understanding non-literal meaning is, thus, a late-acquired language ability. The same can be said of lexical competition, which is the result of increased lexical proficiency, arising from full integration of numerous words in the mental lexicon (Weighall et al. 2017). For instance, 7-year-old children exhibit lexical competition for highly familiar words only, but not adult-like lexical, automatic word recognition (Weighall et al. 2017): after age 7 lexical competition effect increases, specifically for words with higher neighborhood density (Henderson et al. 2013). As mentioned in section 2, older adults tend to surface lexical competition strategies by activating less predictable lexical solutions. Overall, in this respect, aging seems to disrupt again a late-acquired language function.

Simplification of syntactic structures in language production. As also mentioned above, lower memory demands and lexical difficulties account for the syntactic simplification observed in older adults, with particular constraints on embedded utterances and left-stem sentences. During language development, neurotypical children produce complex sentences only after achieving subordinate sentence production (5-year-olds), compound sentence production (3-year-olds) and 2-word sentence production (2-year-olds) (VesserBochane et al. 2020). Cognitive and intellectual development are suggested to be good predictors of complex syntactic production in children, with terminable units (T-unit: main clause + embedded / attached clause) and clausal density (average number of clauses per T-unit) being two robust markers of later syntactic development (Nippold et al. 2005). In this respect, early age-related difficulties with complex syntactic production reverse their late acquisition in childhood. 
Difficulties in understanding complex syntactic structures. Very simple grammatical structures (2-3-word utterances) are usually understood by all children at early ages (Koizumi et al. 2019). However, complex sentence comprehension is extremely variable among children, with success rates correlating with exposure levels to complex syntax, mostly through the parent's speech (Huttenlocher et al. 2002). Children usually achieve a good command on complex syntactic after age 5: later language development allows them to understand how words are arranged into clauses or allocated for creating derived structures, like interrogative or passive sentences (Tolchinsky 2004).

Overall, if we compare age-related milestones in child language development and language changes in healthy aging, we can observe, as summarized in Figure 2, that senescence mainly affects late-acquired language functions, providing support to the hypothesis of language retrogenesis.

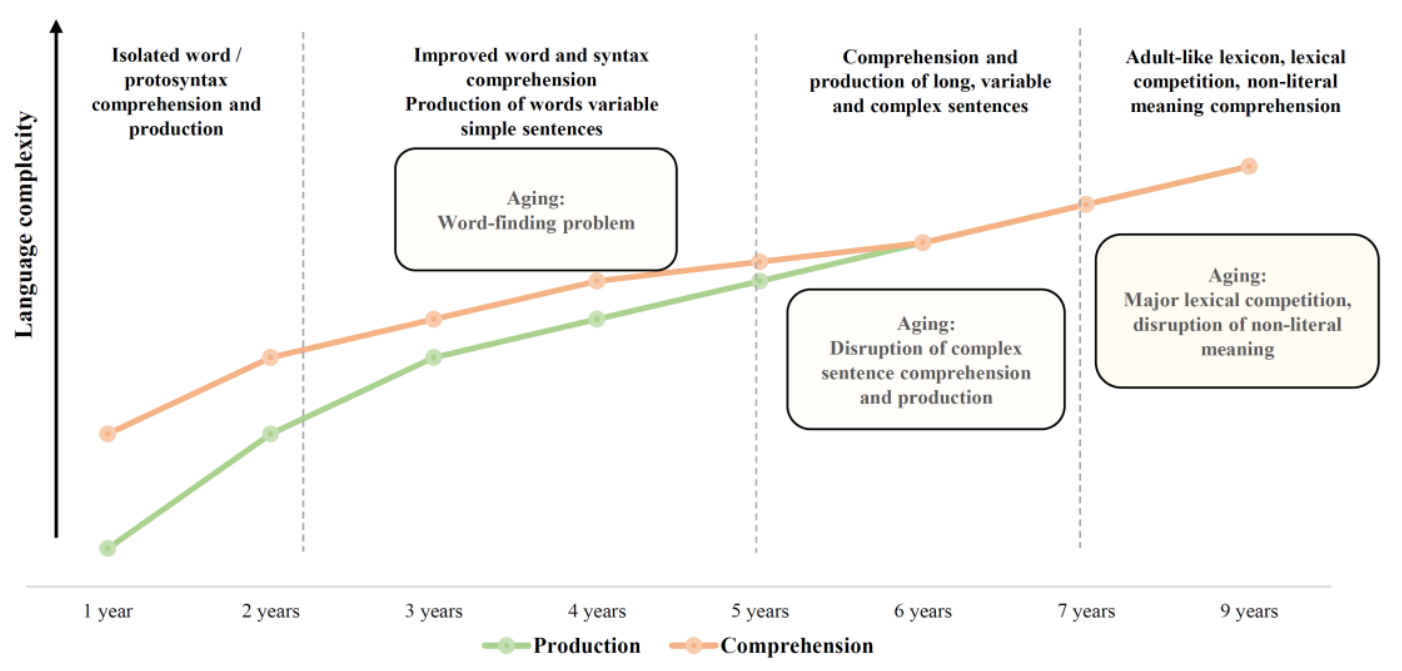

Figure 2. Comparative overlap of language development in neurotypical children and language changes in healthy aging. The figure illustrates some major steps in the development of the child's understanding abilities (in salmon) and production abilities (in green. Colored bubbles suggest inflexion points between late-developing language functions in children and early-disrupted language abilities in healthy aging.

\section{Language retrogenesis and the neurobiological substrate of language}

Although performance scores achieved by healthy elderly in language and cognitive tests aimed to evaluate structural and functional aspects of language seem to give support to 
the language retrogenesis hypothesis, more robust support to this view would result from the consideration of neurobiological evidence. The reason is that brain structure and function are more constrained by the biological factors controlling development and, as such, enable to establish more robust links with evolutionary concerns, in the spirit of EvoDevo theories in biology. Under the retrogenetic view, brain regions/processes that are known to develop/consolidate later during development are expected to be the first ones in being deteriorated/disrupted during aging. A known instance is brain myelination pattern, with the initial progressive disruption of the least myelinated brain regions, which are the last to develop (Reisberg et al. 1999). Likewise, the fronto-temporal areas, which are known to be developed later in life are suggested to be among the first regions to become altered during aging (Rathi et al. 2013).

The aging brain suffers from a number of age-drive processes, like volume reductions in frontal and medial temporal areas (Wingfield and Grossman 2006), mainly in the lateral prefrontal cortex and the hippocampus (Raz et al. 2004). Volume reductions are associated with reduced synaptic density and regional alterations in functional connectivity, including changes in dendritic morphology, neuronal connectivity and neurotransmitter integrity (Wingfield and Grossman 2006). Two age-related processes in the brain stand out for their direct role in the elders' cognitive decline: molecular changes impacting on brain function, like changes in gene methylation patterns (Harman and Martín 2020) or in dopaminergic transmission (Manenti et al. 2013); and structural changes also affecting to brain activity, like reductions in gray matter density, particularly, in the temporal lobe (Fletcher et al. 2018) and the prefrontal cortex (Spreng and Turner 2019), as well as loss of brain white matter (Nilsson et al. 2014).

If we focus on language-related brain areas, these areas present protracted development during the lifespan (Pujol et al. 2006) and, consequently, they undergo late maturation. Initially, language processing depends on functionally-unspecific areas with a more dispersed localization (Messer 2015). Likewise, children show less left lateralization for language (Lidzba et al. 2011), with lateralization to the left occurring with age as a result of increasing activation of left-hemisphere areas associated with language functions (Szaflarski et al. 2006). To some important extent, these features parallel what one finds in the aging brain. First, the aging brain shows a noteworthy neurofunctional dispersion, resulting from an ample reorganization of the neurobiological substrate for cognitive 
functions (Bagarinao et al., 2019). Furthermore, structural differences in the aging brain lead to decline in anterior-posterior gradient affecting late-myelinated languagesupporting areas (left inferior frontal gyrus) in the first instance (Diaz et al. 2016). Parallel decline, in superior-inferior gradient affecting white matter tracts in arcuate fasciculus or superior longitudinal fasciculus III, conveys changes in areas supporting speech production and syntax (Diaz et al., 2016). Overall, in both healthy and pathological aging, more marked changes are observed in late-myelinated brain areas (Gao et al. 2011), suggesting slowdown or breakdown in the cognitive functions they support neuroanatomically (Brickman et al. 2012). Finally, in healthy aging, bilateral activation predominantly substitutes left-lateralized involvement in language processes (Hommet et al. 2008 among others).

Overall, healthy aging results in a functional reorganization of the linguistic brain via structural changes associated to aging that recapitulates, in a reverse way, the last changes occurred in the brain of children. These changes, often described under the general cover term of the Dedifferentiation Hypothesis and, specifically, the Compensation Hypothesis (see Wingfield and Grossman 2006 for a review), suggest that senescence conveys a progressive reduction of cortical specialization for language and the consequent recruitment of other brain areas in language-related tasks. For instance, in order to achieve a good understanding of complex sentences, older adults are observed to involve left dorsal inferior frontal and right temporal-parietal regions (in charge of working memory and grammatical processing of syntax) to compensate for reduced left temporal-parietal activation during sentence understanding (this area is part of the short-term auditoryphonological buffer that enables to maintain information active during sentence processing) (Wingfield and Grossman 2006). Furthermore, when they failed to do so, their brain shows a compensatory activation of dorsolateral prefrontal cortex, which is in charge of general-purpose problem-solving approach to the given task (Wingfield and Grossman 2006). In this way, the most deficient elderly speakers show a significant dedifferentiation in their compensatory brain activation and, thus, a pronounced despecialization. Together, these data suggest that ageing results in more generalized and more basic patterns of brain activation during language processing, resembling those found in young children. 


\section{Language retrogenesis and language evolution}

After showing that available linguistic, cognitive, and neurobiological evidence support to a great extent the language retrogenesis hypothesis, we now address a central issue of our discussion, namely, if language loss in aging might parallel, though inversely, language evolution in the species too. This would eventually mean that the first aspects that are lost during aging, in turn the last traits that are acquired ontogenetically by child language, would be the last features acquired by languages in their phylogenetic complexification. As direct evidence of prehistoric language(s) is lacking, for properly qualifying and testing such a hypothesis, we need to move to the domain of the biological substrate of language, specifically, (paleo)neurology and (paleo)genetics, and examine the available evidence under the light of current theories in evolutionary biology, as noted in the Introduction. We expect this approach to improve past claims based on the consideration of 'degraded' forms of language as proxies, specifically, language in aging.

Seeing it from an ample perspective, the emergence of modern language in humans happened in the context of previously evolved, broader cognitive abilities and behaviors that exhibit a strong evolutionary continuity. As noted by de Waal and Ferrari (2010), the basic building blocks of cognition are shared across a wide range of species. As recently highlighted by Laland and Seed (2021), our species' superior cognitive performance results from interactions and reinforcement at multiple scales between cognitive domains previously evolved. Accordingly, the emergence of present-day languages is expected to have resulted from the refinement of the hominin speech-organs, changes in hominin behavior (particularly, the potentiation of the abilities for cultural niche construction), and subtle modifications of the hominin brain (involving, specifically, the ability for conceptual blending, at the root of language). At the same time, increasing evidence suggests that neurodevelopmental and neurodegenerative risk is mostly linked to humanspecific distinctive features, particularly neurobiological features, including changes in brain anatomy, connectivity, and function (Pattabiraman et al. 2020). Likewise, nearly all genetic variants resulting in increased risk to disease have been found to have humanspecific origins, even though they impact on components that are evolutionarily old (Benton et al. 2021). It is thus useful (and necessary) to contextualize language impairment in aging within our general cognitive and behavioral abilities. One important conclusion of research about language loss in aging is that these abilities, that allow for 
compensatory responses when e.g. working memory impairment impedes language (specifically, word) recovery and use, are not generally interrupted by neither healthy nor pathological aging (see Hodges et al. 1992; Mello et al. 2008). Accordingly, it is specific components of these abilities, or more frequently, the recruitment of these abilities for language that is usually disrupted by aging processes. Having all this mind, we expect specific stages of language loss during aging to be confidently linked to specific stages in the evolution of language in our species, with phylogenetically newer (and, thus, ontogenetically less precocious) brain features exhibiting age-related increased vulnerability to damage, following the rule of 'last-in, first-out' (Raz 2003).

This type of connections has been normally observed in pathological aging. For instance, Douaud and colleagues (2014) identified a brain network that develops late during adolescence and exhibits accelerated degeneration in the elderly, and that encompasses areas of heightened vulnerability in schizophrenia and Alzheimer's disease, like the heteromodal cortex. This network is further associated with cognitive features that have improved in our species, including intellectual ability and episodic memory, and whose impairment contributes to key symptoms of schizophrenia and Alzheimer's dementia. Likewise, Bruner and Jacobs (2013) have linked unique and specialized development (i.e., later species-specific adaptation) of parietal areas involved in higher cognitive functions in humans to vulnerability to neurodegenerative processes and consequent cognitive impairment in humans, particularly, to Alzheimer's disease (Bruner and Jacobs 2013). However, as noted in the Introduction, we think more parsimonious to look for this type of correlations in the neurotypical population, to avoid the possible disturbing influence of the deleterious effects brought about by pathological mutations or brain damage. Available evidence supports the feasibility of a link, specifically, between brain evolution and cognitive disruption in healthy aging resulting from changes in brain structure and function. One example may be taken from the development and understanding/production of passives. Aged people exhibit notable difficulties in processing and understanding passive sentences (Sung et al. 2017), while children begin processing passives only after having developed accurate performance on other structural types, like actives and subject clefts (Dick et al. 2004). Processing of passives relies on the activation of left temporo-occipital regions and bilateral inferior frontal gyrus (IFG), responsible for thematic reanalysis processes and complex syntax processing, respectively (Mack et al. 2013). Dorsal and ventral white matter language pathways 
related to left IFG become more myelinated with age (Wang et al. 2020), with the left IFG being among the late-myelinated cortical areas that are first affected by aging (Diaz et al. 2016). In turn, compared to macaques, the human left IFG shows increased functional integration and coupling, as well as increased myelination, that parallel what is observed during child development (Wang et al., 2020) (see Figure 3 for more details). At the same time, passives are expected to have evolved later than actives, as predicted by the mathematical models of early-step evolution of human language (Nowak and Krakauer 1999). Likewise, Code's (2005) assumption that protolanguage was not lateralized because it might have emerged prior to functional lateralization of the brain finds support in the fact that, as noted above, in healthy aging, bilateral activation predominantly substitutes left-lateralized involvement in language processes. To put another example, the arcuate fascicle, a nervous bundle supporting complex syntax processing, is not (so) developed in non-human primates, is immature in children with syntactic processing problems and is disrupted in patients with lesions deficient in processing complex syntax (Friederici and Gierhan 2013), supporting the view that some late-myelinated areas in childhood and early disrupted areas in aging that involved in language processing are phylogenetically recent.
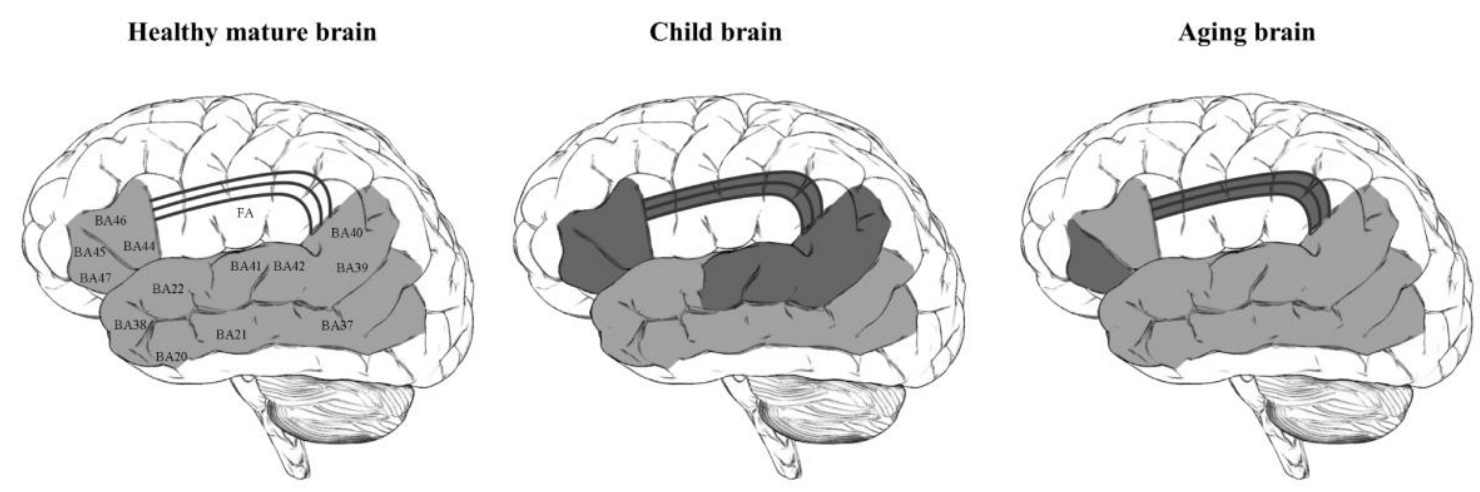

Figure 3. Language-supporting areas in the healthy mature brain, the child's brain and the aging brain. In the healthy mature brain (left), the most prominent language-supporting areas (according to Sakai, 2015 and Ardila et al., 2016) are highlighted in grey. Broca's complex includes left inferior frontal gyrus (F3T/F3O), BA45 and 47; left inferior frontal gyrus, specifically opercular pars and triangular pars, BA44 and 45, and left middle frontal gyrus, BA46. Wernicke's complex includes posterior superior temporal gyrus, BA22; middle temporal gyrus, B21; auditory cortex, BA41 and BA42. Extended Wernicke's area also includes BA20, BA37, BA38, BA39 and BA40. FA (arcuate fasciculus) is considered to be the most important white matter bundle for language network in the brain. In the figure depiciting the language-supporting areas in the healthy child brain (centre), the late-myelinated areas are hyghlighted in dark grey. These include some parts of the Broca's complex areas, related to language production and complex language support, as well as the FA, responsible for integration-production networking. In the figure of the healthy aging brain (right) the darker areas correspond to the language-supporting areas that are more 
susceptible to demyelination. These include the left IFG and the FA. Importantly too, impairments in syntactic and semantic processing in aging, to which the IFG contributes significantly, are hypothesized to be related to the reinforcement of reactive aggression during aging, giving additional support to the view that language-related areas mediate executive abilities involved in the cortical regulation of both aggression and combination, including syntax (Miller et al. 2008).

A final illustrative example of the sort of links we wish to highlight (and that we expect to be informative with regards to language evolution) concerns the cortico-subcortical circuits involved in syntax processing. As Benítez-Burraco and Progovac (2021) describe in detail, language evolution entailed, among other things, the reinforcement of specific cortico-subcortical circuits involved in syntactic chunking. Because these circuits also control reactive aggression, such reinforcement can be hypothesized to have resulted as well in increased prosociality, essential for modern patterns of interaction (and interaction through language). At the same time, the use of increasingly complex forms of language seemingly reinforced the control of subcortical regions by cortical areas, this contributing in turn to reduce reactive aggression. Accordingly, during our evolution, reduced reactive aggression/increased prosociality/increased self-control and sophisticated language are expected to have been engaged in a positive feedback loop, as both aspects are controlled by (and impact on) the same brain circuits that become refined as a consequence of these mutually-reinforcing interaction. Interestingly, older children, with more advanced cognitive development as measured by Theory of Mind, exhibit lower indexes of aggression (Austin et al. 2017). Likewise, increased receptive and expressive language abilities inversely correlate with aggression levels (Ersan 2020). By contrast, children with developmental language disorders show higher scores of aggressive behaviour (Özcebe et al. 2020). Although more pronounced aggression levels are characteristic of pathological aging (dementia), healthy older adults also exhibit both direct and indirect aggression (Ravyts et al. 2021). At the same time, with age, child brain undergoes important developmental functional changes in cortical-subcortical neural circuits (Heller et al. 2016), whereas aging conveys decline in cortico-subcortical connectivity (Grady 2008), suggesting that cognitive functions impairments in aging are due in part to changes in frontal cortical-subcortical networks (see Gawron et al. 2014 for a deep analysis of neuropsychological models of cognitive patterns in healthy elderly). Overall, the increasing control during child development and the decreasing control during in aging over brain areas involved into cognitive control, including selected cortico-subcortical circuits, support such increased language-decreased aggression relationship during language evolution. 


\section{Conclusions and future prospects}

Language acquisition by children and language change/loss in healthy aging generally adjust to the principle of retrogenesis. This is particularly true for complex language tasks, like complex word comprehension and complex syntax comprehension and production. Still, certain asystematicity can be observed in the reversal order of language changes in aging compared to language acquisition by the child, that can be related to age-related experience with language use. For example, while aged people significantly rely on the context for understanding utterances, children only gradually develop abilities to interpret language based on relevant contextual cues (Ryder and Leinonen 2013). Furthermore, linguistic knowledge and procedural rules for language implementation are better trained in the elder and, thus, are more consistent and resistant in aging. Ultimately, in our paper we have shown evidence supporting the view that this retrogenesis can be informative of (and in fact, explained by) evolutionary concerns, with aspects of language more (and earlier) affected in aging being the most recently evolved in our species.

Hypotheses of this sort needs to be properly tested. Clearly, more comparative studies are needed to provide additional support (and to refine) the hypothesis of retrogenesis. We need more data also from non-WEIRD societies, as the cultural and sociological environments in which people grow and age can differ significantly: as we have discussed above, this environment is expected to have a deep impact on language acquisition and language loss. Likewise, we need to go on delving into the psycholinguistics aspects of language acquisition by the child and language variation in the elderly, as this approach is expected to provide a clearer picture of the cognitive aspects involved, but also to facilitate a more precise neurobiological account of language acquisition and language loss, of the sort we have sketched in section 4 above. Genetic studies could help us as well to know more about the biological causes of the observed variation when acquiring or losing a language. In turn, better neurobiological and genetic characterizations of language in aging are the key to conduct comparative studies in other species, aimed to refine our knowledge of the evolutionary trajectory of the language-related areas that are differentially impacted by aging. Eventually, studies using ancient DNA might result in a more detailed account of these evolutionary changes, particularly, of gene modifications important for explaining language changes in aging, because of the deep connection between evolution and development, of the sort we have highlighted in the paper. 


\section{References}

Abrams, L. and M.T. Farrell (2010). Language processing in normal aging. In J. Guendouzi, F. Loncke and M.J. Williams (eds.), The Handbook of Psycholinguistic and Cognitive Processes, pp. 49-74. New York/London: Psychology Press.

Antonenko, D., J. Brauer, M. Meinzer, A. Fengler, L. Kerti, A.D. Friederici and A. Flöel (2013). Functional and structural syntax networks in aging. NeuroImage 83, 513-523.

Ardila, A. (2006). Orígenes del lenguaje: un análisis desde la perspectiva de las afasias. Revista de Neurología 43(11), 690-698.

Ardila, A. (2015). A proposed neurological interpretation of language evolution. Behavioural Neurology (online).

Ardila, A., B. Bernal and M. Rosselli (2016). How localized are language brain areas? A review of Brodmann areas involvement in oral language. Archives of Clinical Neuropsychology 31, 112-122.

Austin, G., R. Bondü and B. Elsner (2017). Longitudinal relations between children's cognitive and affective theory of mind with reactive and proactive aggression. Aggressive behavior 43(5), 440-449.

Bagarinao, E., H. Watanabe, S. Maesawa, D. Mori, K. Hara, K. Kawabata et al. (2019). Reorganization of brain networks and its association with general cognitive performance over the adult lifespan. Scientific Reports 9(1): 11352.

Bambini, V., L. van Looy, K. Demiddele and Q. Schaeken (2021). What is the contribution of executive functions to communicative-pragmatic skills? Insights from aging and different types of pragmatic inference. Cognitive Processing. https://doi.org/10.1007/s10339-021-01021-w 
Barresi, B.A., M. Nicholas, L. Tabor Connor, L.K. Obler and M.L. Albert (2000). Semantic degradation and lexical access in age-related naming failures. Aging, Neuropsychology, and Cognition 7(3), 169-178.

Bates, E. and F. Dick (2002). Language, gesture, and the developing brain. Developmental Psychobiology 40(3), 293-310.

Benítez-Burraco, A. and C. Boeckx (2014). Language disorders and language evolution: constraints on hypotheses. Biological Theory 9, 269-274.

Benton, M.L., A. Abraham, A.L. LaBella, P. Abbot, A. Rokas and J.A. Capra (2021). The influence of evolutionary history on human health and disease. Nature Reviews Genetics 22, 269-283.

Botha, R. (2006). On the windows approach to language evolution. Language and Communication 26(2), 129-143.

Brickman, A.M., I.B. Meier, M.S. Korgaonkar, F.A. Provenzano, S.M. Grieve, K.L. Siedlecki, B.T. Wasserman, L.M. Williams and M.E. Zimmerman (2012). Testing the white matter retrogenesis hypothesis of cognitive aging. Neurobiology of Aging 33(8), 1699-1715.

Bruner, E. and H.I.L. Jacobs (2013). Alzheimer's disease: the downside of a highly evolved parietal lobe? Journal of Alzheimer's Disease 35(2), 227-240.

Burke, D.M and D.G. Mackay (1999). Memory, language, and ageing. Philosophical Transactions of the Royal Society of London. Series B: Biological Sciences 352 (1363), 1845-1856.

Burke, D.M., D.G. MacKay and L.E. James (2000). Theoretical approaches to language and aging. In T. J. Perfect and E. A. Maylor (Eds.), Debates in psychology. Models of cognitive aging, pp. 204-237. Oxford: Oxford University Press. 
Burke, D.M. and E. R. Graham (2012). The neural basis for aging effects on language. In M. Faust (ed.), The Handbook of the Neuropsychology of Language, pp. 774-796. Chichester: Wiley Blackwell.

Caplan, D., G. DeDe, G. Waters, J. Michaud and Y. Tripodis (2011). Effects of age, speed of processing, and working memory on comprehension of sentences with relative clauses. Psychology and Aging 26(2), 439-450.

Clark, D.G. and J.L. Cummings (2003). Aphasia. In T. Brandt, L.R. Caplan, J. Dichgans, H.C. Diener \& C. Kennard (eds.), Neurological Disorders, 265-275. Amsterdam et al.: Academic Press.

Code, C. (2005). First in, last out?: The evolution of aphasic lexical speech automatisms to agrammatism and the evolution of human communication. Interaction Studies 6(2), 311-334.

Code, C. (2011). Nonfluent aphasia and the evolution of proto-language. Journal of Neurolinguistics 24(2), 136-144.

Cohen, R.A., M.M. Marsiske and G.E. Smith (2019). Neuropsychology of aging. In Handbook of Clinical Neurology 167, 149-180.

De Boer, B. (2019). Evolution of speech: anatomy and control. Journal of Speech, Language and Hearing Research, 62(8S), 2932-2945.

DeDe, G. and J. Knilans Flax (2016). Language comprehension in aging. In H.H. Wright (ed.), Cognition, Language and Aging, 107-134. Amsterdam/Philadelphia: John Benjamins.

De Waal, F. and P.F. Ferrari (2010) Towards a bottom-up perspective on animal and human cognition. Trends in Cognitive Sciences 14, 201-207. 
Diaz, M.T., A.A. Rizio and J. Zhuang (2016). The neural language systems that support healthy aging: integrating function, structure, and behavior. Language and Linguistics Compass 10(7), 314-334.

Dick, F., B. Wulfeck, M. Krupa-Kwiatkowski and E. Bates (2004). The development of complex sentence interpretation in typically developing children compared with children with specific language impairments or early unilateral focal lesions. Developmental Science 7(3), 360-377.

Dixon, J.A. and V.A. Marchman (2007). Grammar and the lexicon: developmental ordering in language acquisition. Child Development 78(1), 190-212.

Douaud, G., A.R. Groves, C.K. Tamnes, L.T. Westlye et al. (2014). A common brain network links development, aging, and vulnerability to disease. PNAS 49, 17648-17653.

Emery, V.O.B. (2000). Language impairment in dementia of the Alzheimer type: a hierarchical decline? The International Journal of Psychiatry in Medicine 30(2), 145-164.

Ersan, C. (2020). Early language development and child aggression. World Journal of Education 10(1), 1-11.

Fitch, W.T. (2000). The evolution of speech: a comparative review. Trends in Cognitive Science 4(7), 258-267.

Fitch, W.T. (2017). Preface to the Special Issue on the biology and evolution of language. Psychonomic Bulletin and Review 24, 1-2.

Fletcher, E., B. Gavett, D. Harvey, S. Tomaszewski Farias, J. Olichney, L. Beckett, C. DeCarli and D. Mungas (2018). Brain volume change and cognitive trajectories in aging. Neuropsychology 32(4), 436-449.

Friederici, A. and S.M.E. Gierhan (2013). The language network. Current Opinion in Neurobiology 23(2), 250-254. 
Gao, J., R.T.F. Cheung, T.M.C. Lee, L.W. Chu, Y.S. Chan, H.K.F. Mak, J.X. Zhang, D. Qiu, G. Fung and C. Cheung (2011). Possible retrogenesis observed with fiber tracking: an anteroposterior patterns of white matter disintegrity in normal aging and Alzheimer's disease. Journal of Alzheimer's Disease 26(1), 47-58.

Gayraud, F., I. Simoes Loureiro, C. Frouin, C. Collette, L. Lefebvre and M. BarkatDefradas (2019). Lexical deterioration in Alzheimer's disease. ExLing 2019: Proceedings of $10^{\text {th }}$ International Conference of Experimental Linguistics, 112-115.

Gawron, N., E. Lojek, B. Kijanowska-Haladyna, J. Nestorowicz, A. Harasim, A. Pluta and M. Sobanska (2014). Cognitive patterns of normal elderly subjects are consistent with frontal cortico-subcortical and fronto-parietal neurpsychological models of brain aging. Applied Neuropscyhology: Adult 21(3), 195-209.

Glisky, E.L. (2007). Changes in cognitive function in human aging. In D.R. Riddle (ed.), Brain aging: models, methods, and mechanisms. Boca Raton: CRC Press.

Gollan, T.H. and M. Goldrick (2019). Aging deficits in naturalistic speech production and monitoring revealed through reading aloud. Psychology and Aging 34(1), 25-42.

Grady, C.L. (2008). Cognitive neuroscience of aging. Annals of the New York Academy of Sciences 1124, 127-144.

Harada, C.N., M.C. Natelson Love and K.L. Triebel (2013). Normal cognitive aging. Clinics in Geriatric Medicine 29(4), 737-752.

Hardy, S. M., K. Messenger and E.A. Maylor (2017). Aging and syntactic representations: Evidence of preserved syntactic priming and lexical boost. Psychology and Aging 32(6), 588-596.

Hardy, S.M., K. Segaert and L. Wheeldon (2020). Healthy aging and sentence production: disrupted lexical access in the context of intact syntactic planning. Frontiers in Psychology 11: 257. 
Harman, M.F. and M.G. Martín (2020). Epigenetic mechanisms related to cognitive decline during aging. Journal of Neuroscience Research 98(2), 234-246.

Heller, A.S., A.O. Cohen, M.F.W. Dreyfuss and B.J. Casey (2016). Social Cognitive and Affective Neuroscience, 1910-1918.

Henderson, L., A. Weighall, H. Brown and G. Gaskell (2013). Online lexical competition during spoken word recognition and word learning in children and adults. Child Development 84(5), 1668-1685.

Hodges, J.R., D.P. Salmon and N. Butters (1992). Semantic memory impairment in Alzheimer's disease: failure of access or degraded knowledge? Neuropsychologia 30(4), 301-314.

Hommet, C., C. Destrieux, T. Constans \& G. Berrut (2008). Influence de l'âge sur la latéralisation cérébrale. Psychologie \& NeuroPsychiatrie du vieillissement 6(1), 49-56.

Huttenlocher, J., M. Vasilyeva, E. Cymerman and S. Levine (2002). Language input and child syntax. Cognitive Psychology 45(3), 337-374.

Jackendoff, R. (1999). Possible stages in the evolution of the language capacity. Trends in Cognitive Sciences 3(7), 272-279.

Juncos-Rabadán, O., D. Facal, M.S. Rodríguez and A.X. Pereiro (2010). Lexical knowledge and lexical retrieval in ageing: insights from a tip-of-the tongue (TOT) study. Language and Cognitive Processes 25(10), 1301-1334.

Kempler, S., M. Thompson and J. Marquis (2001). Longitudinal change in language production: effects of aging and dementia on grammatical complexity and propositional content. Psychology and Aging 16(4), 600-614.

Kempler, S., R.E. Herman and C.J. Liu (2004). Sentence production by young and older adults in controlled contexts. The Journal of Gerontology Series B: Psychological Sciences and Social Sciences 59, 220-224. 
Kim, H., J.H. Yoon, J.E. Lee, E.J. Baek, Y.H. Sohn and D.L. Na (2011). Is confrontation naming performance in Alzheimer's Disease the nominal linguistic retrogenesis of normal development? European Neurology 66(4), 195-199.

Koizumi, M., Y. Saito and M. Kojima (2019). Syntactic development in children with intellectual disabilities - using structured assessment of syntax. Journal of Intellectual Disability Research 63(12), 1428-1440.

Laland K \& A. Seed (2021). Understanding human cognitive uniqueness. Annual Review of Psychology 72, 689-716.

Leal, S.L. and M.A. Yassa (2019). Normal cognitive and brain aging. In M.L. Alosco and R.A. Stern (eds.), The Oxford Handbook of Adult Cognitive Disorders, pp. 5-27. Oxford: Oxford University Pres.

Lee, C.L. and K.D. Federmeier (2010). Differential age effects on lexical ambiguity resolution mechanisms. Psychophysiology 48(7), 960-972.

Lidzba, K., E. Schwilling, W. Grodd, I. Krägeloh-Mann and M. Wilke (2011). Language comprehension vs. language production: age effects of fMRI activation. Brain and Language 119(1), 6-15.

Luinge, M.R., W.J. Post, J.P. Wit and S.M.Goorhuis-Brouwer (2006). The ordering of milestones in language development for children from 1 to 6 years of age. Journal of Speech, Language and Hearing Research 49(5), 923-940.

Mack, J.E., A. Meltzer-Asscher, E. Barbieri and C.K. Thompson (2013). Neural correlates of processing passive sentences. Brain Sciences 3(3), 1198-1214.

Manenti, R., M. Brambilla, M. Petesi, C. Miniussi and M. Cotelli (2013). Compensatory networks to counteract the effects of ageing on language. Behavioural Brain Research 249, 22-27. 
Mattys, S. L., and O. Scharenborg (2014). Phoneme categorization and discrimination in younger and older adults: A comparative analysis of perceptual, lexical, and attentional factors. Psychology and Aging, 29(1), 150-162.

Mello, C.B. de, K. Abrisqueta-Gomez, G.F. Xavier and O.F. Amodeo Bueno (2008). Involution of categorical thinking processes in Alzheimer's disease: preliminary results. Dementia and Neuropsychologia 2(1), 57-62.

Melvold, J.L., Au, R., Obler, L.K. and Albert, M.L. (1994). Language during aging and dementia. In M.L. Albert and J.E. Knoefel (eds.), Clinical Neurology of Aging (pp. 329346). New York / Oxford: OUP.

Messer, D. and J. Dockrell (2006). Children's naming and word-finding difficulties: descriptions and explanations. Journal of Speech, Language and Hearing Research 49(2), 309-324.

Messer, R.H. (2015). Pragmatic language changes during normal aging: implications for health care. Healthy Aging and Clinical Care in the Elderly 7, 1-7.

Miller, L. A., Collins, R. L. and Kent, T. A. (2008). Language and the modulation of impulsive aggression. Journal of Neuropsychiatry and Clinical Neuroscience 20(3), 26173. doi: 10.1176/jnp.2008.20.3.261.

Newman, R.S., D.J. German and J.R. Jagielko (2018). Influence of lexical factors on word-finding accuracy, error patterns, and substitution types. Communication Disorders Quarterly 39(2), 356-366.

Nilsson, J., A.J. Thomas, J.T. O'Brien and P. Gallagher (2014). White matter and cognitive decline in aging: a focus on processing speed and variability. Journal of the International Neuropsychological Society 20, 262-267.

Nippold, M.A., L.J. Hesketh, J.K. Duthie and T. Mansfield (2005). Conversational versus expository discourse. A study of syntactic development in children, adolescents, and adults. Journal of Speech, Language and Hearing Research 48(5), 1048-1064. 
Nowak, M.A. and D.C. Krakauer (1999). The evolution of language. PNAS 96, 80288033.

Ouyang, M., X. Cai and Q. Zhang (2020). Aging effects on phonological and semantic priming in the tip-of-the-tongue: evidence from a two-step approach. Frontiers in Psychology 11.

Özcebe, E., A. Noyan Erbas and T. Karahan Tigrak (2020). Analysis of behavioural characteristics of children with developmental language disorders. International Journal of Speech-Language Pathology 22(1), 30-36.

Pattabiraman K, Muchnik SK, Sestan N (2020) The evolution of the human brain and disease susceptibility. Current Opinion in Genetics and Development 65, 91-97. doi: 10.1016/j.gde.2020.05.004.

Peelle, J. (2019). Language and aging. In G.I. de Zubicaray and N.O. Schiller (eds.), The Oxford Handbook of Neurolinguistics, pp. 295-316. Oxford: OUP.

Pistono, A., L. Guerrier, P. Péran, M. Rafiq, M. Giméno, C. Bézy, J. Pariente and M. Jucla (2021). Increased functional connectivity supports language performance in healthy aging despite gray matter loss. Neurobiology of Aging 98, 52-62.

Poulisse, C., L. Wheeldon and K. Segaert (2019). Evidence against preserved syntactic comprehension in healthy aging. Journal of Experimental Psychology: Learning, Memory, and Cognition 45(12), 2290-2308. Doi: https://doi.org/10.1037/xlm0000707

Pujol, J., C. Soriano-Mas, H. Ortiz, N. Sebastián-Gallés, J.M. Losilla and J. Deus (2006). Myelination of language-related areas in the developing brain. Neurology 66, 339-343.

Rabaglia, C.D. and T.A. Salthouse (2011). Natural and constrained language production as a function of age and cognitive abilities. Language and Cognitive Processes 26(10), 1505-1531. 
Rathi, Y., O. Pasternak, P. Savadjiev, O. Michailovich, S. Bouix, M. Kubicki, C.-F. Westin, N. Makris and M.E. Shenton (2013). Gray matter alterations in early aging: a diffusion magnetic resonance imaging study. Human Brain Mapping 35(8), 3841-3856.

Ravyts, S.C., E. Perez, E.K. Donovan, P. Soto and J.M. Dzierzewski (2021). Measurement of aggression in older adults. Aggression and Violent Behavior 57, 101484.

Raz, N. (2003). Ageing and the Brain. Encyclopedia of Life Sciences. Accesible online: https://onlinelibrary.wiley.com/doi/full/10.1038/npg.els.0003375

Raz, N., K.M. Rodrigue, D. Head, K.M. Kennedy and J.D. Acker (2004). Differential aging of the medial temporal lobe. A study of five-year change. Neurology 62(3), 433439.

Reisberg, B., E.H. Franssen, S.M. Hasan, I. Monteiro, I. Boksay, L.E.M. Souren, S. Kenowsky, S.R. Auer, S. Elahi and A. Kluger (1999). Retrogenesis: clinical, physiologic, and pathologic mechanisms in brain aging, Alzheimer's and other dementing processes. European Archives of Psychiatry and Clinical Neuroscience 249, 28-36.

Roemer, E.J., K.L. West, J.B. Northrup and J.M. Iverson (2019). Word comprehension mediates the link between gesture and word production: examining language development in infant siblings of children with autism spectrum disorder. Developmental Science 22(3): e12767.

Rubial-Álvarez, S., S. de Sola, M.C. Machado, E. Sintas, P. Böhm, G. SánchezBenavides, K. Langohr, R. Muñiz and J. Peña-Casanova (2013). The comparison of cognitive and functional performance in children and Alzheimer's disease supports the retrogenesis model. Journal of Alzheimer's Disease 33, 191-203.

Ryder, N. and E. Leinonen (2013). Pragmatic language development in language impaired and typically developing children: incorrect answers in context. Journal of Psycholinguistic Research 43, 45-58. 
Sakai, K.L. (2015). Language acquisition and brain development. Science 310, 815-819

Shafro, M.A., D.M. Burke, E.A. Stamatakis, P.P. Tam and L.K. Tyler (2007). On the tipof-the-tongue: neural correlates of increased word-finding failures in normal aging. Journal of Cognitive Neuroscience 19(12), 2060-2070.

Shafro, M.A. and L.K. Tyler (2014). Language in the aging brain: the network dynamics of cognitive decline and preservation. Science 346(6209), 583-587.

Simoes Loureiro, I. and L. Lefebvre (2016). Retrogenesis of semantic knowledge. Comparative approach of acquisition and deterioration of concepts in semantic memory. Neuropsychology 30(7), 853-859.

Slobin, D.I. (2005). From ontogenesis to phylogenesis: what can child language tell us about language evolution? In S.T. Parker, J. Langer and C. Milbrath (eds.), Biology and Knowledge Revisited: from Neurogenesis to Psychogenesis, pp. 255-286. New York / London: Psychology Press.

Spreng, R.N. and G.R. Turner (2019). The shifting architecture of cognition and brain function in older adulthood. Perspectives on Psychological Science 14(4), 523-542.

Steels, L. and E. Szathmáry (2018). The evolutionary dynamics of language. Biosystems $164,128-37$.

Sundaray, S., T. Marinis and A. Bose (2018). Comprehending non-literal language: effects of aging and bilingualism. Frontiers in Psychology 9: 2230.

Sung, J.E., J.K. Yoo, S.E. Lee and B. Eom (2017). Effects of age, working memory, and word order on passive-sentence comprehension: evidence from a verb-final language. International Psychogeriatrics 29(6), 939-948.

Szaflarski, J.P., V.J. Schmithorst, M. Altaye, A.W. Byars, J. Ret, E. Plante and S.K. Holland (2006). A longitudinal functional magnetic resonance imaging study of language development in children 5 to 11 years old. Annals of Neurology 59(5), 796-807. 
Tolchinsky, L. (2004). The nature and scope of later language development. In R.A. Berman (ed.), Language Development across Childhood and Adolescence, 233-248. Amsterdam/Philadelphia: John Benjamins.

Tremblay, P., I. Deschamps, P. Bédard, M.-H. Tessier, M. Carrier and M. Thibeault (2018). Aging of speech production, from articulatory accuracy to motor timing. Psychology and Aging 33(7), 1022-1034.

Tucker, B.V., C. Ford and S. Hedges (2020). Speech aging: production and perception. WIREs Cognitive Science: e1557.

Uekermann, J., P. Thoma and I. Daum (2008). Proverb interpretation changes in aging. Brain and Cognition 67(1), 51-57.

Vasilyeva, M., H. Waterfall and J. Huttenlocher (2007). Emergence of syntax: commonalities and differences across children. Developmental Science 11(1), 84-97.

Venneri, A., C. Jahn-Carta, M. de Marco, D. Quaranta and C. Marra (2018). Diagnostic and prognostic role of semantic processing in preclinical Alzheimer's disease. Biomarkers in Medicine 12(6), 637-651.

Wang, J., Y. Yang, X. Zhao, Z. Zuo and L.H. Tan (2020). Evolutional and developmental anatomical architecture of the left inferior frontal gyrus. NeuroImage 222, 117-268.

Weighall, A.R., L.M. Henderson, D.J. Barr, S.A. Cairney and M.G. Gaskell (2017). Eyetracking the time-course of novel word learning and lexical competition in adults in children. Brain and Language 167, 13-27.

Wingfield, A. and M. Grossman (2006). Language and the aging brain: patterns of neural compensation revealed by functional brain imaging. Journal of Neurophysiology 96, 2830-2839. 
Wingfield, A., N.M. Amichetti and A. Lash (2015). Cognitive aging and hearing acuity: modeling spoken language comprehension. Frontiers in Psychology 6: 684.

Wlotko, E.W., C.L. Lee and K.D. Federmeier (2010). Language of the aging brain. Eventrelated potential studies of comprehension in older adults. Language and Linguistics Compass 4(8), 623-638. 